\title{
Designing Search Engine User Interfaces for the visually impaired
}

\author{
Barbara Leporini \\ ISTI, National Research Council \\ via Moruzzi, 1 \\ 56010 Pisa, Italy \\ +39-050-3152034 \\ Barbara.Leporini@isti.cnr.it
}

\author{
Patrizia Andronico \\ IIT, National Research Council \\ via Moruzzi, 1 \\ 56010 Pisa, Italy \\ +39-050-3152090 \\ Patrizia.Andronico@iit.cnr.it
}

\author{
Marina Buzzi \\ IIT, National Research Council \\ via Moruzzi, 1 \\ 56010 Pisa, Italy \\ $+39-050-3152631$ \\ Marina.Buzzi@iit.cnr.it
}

\begin{abstract}
Search engines are a fundamental tool for retrieving specific and appropriate information on the Internet; for this reason it is essential for any user to be able to interact with simple, clear and accessible interfaces. In this paper we describe the main design issues affecting the user interface of a search engine when a sightless user interacts by means of a screen reader or voice synthesizer. In particular, the most important differences between a visual layout and aural perception are discussed, in order to propose appropriate and specific guidelines for improving the design of search engine interfaces.
\end{abstract}

\section{Categories and Subject Descriptors}

H.5.2 [Information Interfaces and Presentation]: User Interfaces - User-centered design

\section{General Terms}

Search engine, user interface, Internet, web navigation.

\section{Keywords}

Search engine, user interface design, accessibility, usability.

\section{INTRODUCTION}

The enormous amount of data available today on the World Wide Web requires the use of search tools for retrieving useful information. The search and retrieval of information is important for everyone but it is crucial for people with disabilities, especially for the blind, who cannot access printed information. Computers and the Internet have significantly contributed to the increased independence of disabled people in study, work, and free time, leading to greater autonomy and social integration. For this reason it is critical to make search tools universally accessible and easy to use for all, particularly for visually-impaired and blind users who interact by means of assistive devices (such as screen readers) and have more difficulties than sighted users. This work is based on preliminary testing of accessibility and usability of different search tools (search engine, directories and metasearch engines); testing involved the use of automatic tools (validators) and a survey [1]. A questionnaire was distributed to both sighted and sightless users in order to collect and analyze feedback on search engines; the complete results and discussion

Copyright is held by the author/owner(s).

W4A at WWW2004, May 18, 2004, New York, New York, USA.

Copyright 2004 ACM 1581139039/04/0005 . . \$5.00. of the survey are found in [2]. The questionnaire was organized into four sections: user description (age, sex, ICT skill); general knowledge of search tools; use of search engine options (advanced search, refinement function and preferences); difficulties using search tools. The survey showed that only $25 \%$ of the sample configured the search engine (i.e. search options, preferences) while $75 \%$ performed the advanced search at least once; $38 \%$ of these were blind and $87 \%$ sighted. Usually users formulated queries with more than one keyword ( $92 \%$ of sighted and $69 \%$ of blind users) and $67 \%$ of sighted users had no difficulty choosing the right words, whereas this was true only for $38 \%$ of sightless. It is remarkable that $92 \%$ of sighted users thought that search engines are easy to use but less than $7 \%$ of blind users agree. In fact, the sighted can rapidly select interesting results or discard irrelevant information, whereas it takes longer for blind users due to the serial access to web page content. Concerning results, a total of $67 \%$ of sighted users could explore more than two pages with respect to $15 \%$ of blind users. Only $23 \%$ of blind users used the refining function (for searching within results) compared to $59 \%$ of sighted users. Furthermore, the presence of sponsored results was known to $48 \%$ of the subjects, but only $25 \%$ were able to identify them. For sighted users the main obstacle was choosing the right keywords $(62 \%)$ while blind users also had difficulty reading results $(46 \%$ compared to $15 \%$ of sighted users) and accessing interfaces (functions/interface unclear). Last, the survey highlighted that only $38 \%$ of blind users were able to find useful information compared to $90 \%$ of sighted users. These data demonstrate that user interface (UI) design has great impact on sightless users.

In this paper we discuss the main differences between a visual layout and aural perception, and propose a set of guidelines for designing search engine UIs. The following is organized into four sections: first, related works available in the literature are described; next, we discuss issues in web navigation for sightless users when interacting with a screen reader and voice synthesizer. In the third section the main differences between a visual layout and aural perception are illustrated, along with an example of a popular search engine. Lastly, we indicate a set of guidelines for improving usability of search engine UIs.

\section{Related Works}

There are many studies dedicated to user-interface accessibility and usability, but few focus on search engine interfaces. Detailed usability guidelines have been formulated for user interfaces and Web page design in [11] and [12]. Unfortunately, search engines are particularly difficult for the blind to use, since problems in Web navigation add to the complexity of the search engine interface and functions. In [8] and [9], a possible combination of 
usability and accessibility criteria for the visually-impaired is proposed, since both are necessary for those who need assistive devices to browse the Internet. Regarding accessibility, the W3C Consortium is one of the main sources of information, tools and resources. Within the framework of the Web Accessibility Initiative (WAI) the W3 Consortium proposed a set of 14 main guidelines [14]. In addition, other accessibility guidelines have been defined by section 508 standards [15]. A specific study concerning accessibility of on-line library resources was carried out in [13] while an auditory search engine prototype, providing vocal output by using real-time text-categorization to organize results into a voice menu format, is discussed in [6]. The NOVA project, sponsored by the Manchester Metropolitan University studies the usability and accessibility problems of the sightless and visually-impaired when retrieving information, with particular focus on access to Digital Library systems. Specifically, usability experiments were carried out on a sample of blind and visually-impaired individuals who performed four informationseeking tasks, including the use of search engines. Interesting and detailed results are included in [4]. Last, in [3] the author discusses the limits of universal design and analyzes the use of a text-transcoder to furnish a text interface equivalent to the original. In particular, for people using a screen reader (which gives modal access) user bandwidth decreases (e.g. actions take longer) while tasks become more difficult since additional actions are required. Therefore, when designing accessible interfaces it is very important to maximize the "quality of use" for disabled persons. This work describes the initial results of a study on search engine user interface accessibility and usability. In fact, it is very important to consider user needs from the very beginning of the design phase of the interface. In the following, we first introduce some difficulties encountered when navigating by screen reader.

\section{INTERNET NAVIGATION FOR BLIND USERS}

Usually the analysis of digital obstacles for the disabled only addresses accessibility, although usability is fundamental for simplifying both navigation and interaction for users using assistive devices or those with special needs. Blind users navigate the Internet by using a screen reader, which announces the page content, whereas visually-impaired users can interact by means of magnifying programs. Although this study only considers blind users, it is important to note that both user typologies share many navigational difficulties. The main problems for a blind person navigating via screen reader are:

- Lack of context - Navigating by screen reader (or a magnifier) the user accesses only small portions of texts and may lose the overall context of the current page.

- Information overload - The unchanging portions of the site (menu, frames with banners, etc.) may overload the reading, because the user has to read through all the items nearly every time, thus slowing down navigation.

- Excessive sequencing in reading the information - The command for navigating and reading can force the user to follow the page content sequentially. This may provoke great frustration in the user.

- Keyboard navigation - Blind users do not use the mouse function (i.e. pointing, scrolling, selecting, etc.) for moving around the page; but instead move by means of keyboard commands, such as Tab key, arrow keys, and so on. Consequently, navigation around a page is slowed.

- Screen reader interpretation - The screen reader deals with Web page content in a manner that differs greatly from visual rendering. This requires a certain expertise in advanced screen reader and browser commands and orientation oneself within the page content can require considerable effort.

Thus, although the Internet is a precious source of information and offers great availability of services (e-learning, e-business, egovernment, etc.) all these drawbacks can discourage blind and visually-impaired users from accessing on-line services

\subsection{Search Engine Interfaces}

The user interface plays a crucial role in the correct and productive use of a search engine. It is not sufficient for the interface to be accessible - it must also be user-friendly, i.e. easy to use and navigate by all, regardless of devices used. Accessibility guarantees use to anyone; accessible design ensures graceful transformation, as well as understandable and navigable content. Usability renders Internet navigation more effective, efficient and satisfactory. A user interface is composed of many features such as:

- Arrangement of components. This point is quite relevant since value-enhancing features are more "visible" when located in an area that is rapidly encountered by eye movement and does not require page scrolling. In the case of the visually-impaired the most relevant features/functions should be placed in a "relevant" position which means at the top of the page or in an easily-reached point.

- Expressive power: a visual representation can communicate certain kinds of information much more rapidly and effectively than other methods [10]. For this reason the interface design should try to maintain the same degree of expression in both the visual and aural versions.

- Number of elements. Simplicity helps unskilled users navigate the interface easily while an interface full of elements can create confusion and waste time. For instance, for a blind user it is very difficult to use a web directory/portal for searching, due to the complexity of its home page.

- Functions. A user typically performs a simple search and specifies one or more words, obtaining a large set of results. Further criteria selection can be specified in order to restrict search results. Preferences and commands, although very powerful, are rarely used, even by skilled individuals.

- Clustering permits users to explore results grouped by categories. In this way users can navigate a single branch of results more efficiently. This feature, if correctly implemented (i.e. accessible), renders an interface usable for the disabled, and saves time when exploring the search engine output.

Some specific features which can facilitate interaction between blind users and interfaces are:

- Simplicity - It is important that the interface be simple. Fields, combo-boxes, and "search" buttons should be located 
at the top of the page; no advertising banner frames, links, or text should be placed before search fields and results.

- $\quad$ Labelling fields - Using appropriate tags for assigning a label to the associated field is very important in order for the screen reader to recognize it easily. Also, labels should be placed above or to the left of the field, and not below; otherwise, this could create some confusion when using arrow keys to explore the page. The text used for labels and buttons should be simple and familiar.

- $\quad$ Rapid access - Navigation and positioning over main fields and results is faster if access keys and tabindex values are defined. By means of shortcuts and "priority values" users are able to reach the desired search fields or result links more quickly.

- Navigation links - Links or buttons "Next" and "Previous" are particularly important when navigating by keyboard. However, in order to make them really efficient, it is necessary to assign them an access key (e.g., alt $+n$ and alt $+p$ ). In this way, users are able to move rapidly among result pages. Furthermore, links to next result pages should be placed at the end of the page, because, when a user interacts by Tab key, it is frustrating to hear the voice synthesizer recite all these links, before getting to others more interesting parts.

- Layout of search results - Search results are the basic component of a search engine. It is very important to structure them appropriately, so that users are able to read them quickly and easily. Therefore, in order to facilitate navigation by screen reader and keyboard commands, specific features and an adequate structure should be applied. Reaching the result area quickly, clearly knowing the number of results obtained, skipping over a result to the next, and so on, are important features for users who perceive the information by means of a voice synthesizer or Braille display.

In order to reduce the "digital divide" the design of every interface should be user-centered, considering the special needs of the disabled. In general, redesigning an existing site can be onerous in the case of large, dynamic sites, but for search engines, which have at most four interfaces (simple search, advanced search, results and preferences) the cost is low and benefits are considerable.

\section{VISUAL LAYOUT VS AURAL PERCEPTION}

For users interacting by means of assistive technologies, the UI layout and structure are crucial. When navigating by screen reader the user perceives page content which differs from its rendering on the screen. In this section, we first discuss the main differences between visual rendering and aural perception; we then present an example of a search engine interface, including a string search.

\subsection{Visual and Aural Perception}

For understanding differences between visual rendering (with mouse interaction) and aural navigation with keyboard interaction, it is fundamental to know how the screen reader deals with web contents. The screen reader referred to in the following is JAWS for Windows [7], [5], commonly used by blind people all over the world. JAWS provides access to page content and information about its structure and organization. HTML tags in fact allow screen readers to provide detailed information about web page structure: tables, lists, headings, and so forth are all announced. In brief, the screen reader gets the page source, parses the HTML code and serializes the content in the same order as it appears in the source. Therefore, links, text, DIV blocks, frames, etc. are concatenated in sequential order as a single column. Consequently, blind users perceive the page content like a text document: they can read line by line (by arrow keys), or link by link (by Tab key). However, other specific screen reader commands are available (e.g. "t" for skipping to the next table, " $h$ " for moving to the next heading, and so on). From this point of view, many typical visual features are not perceived, but others, such as hidden labels, can be used. Then, information provided by the visual rendering should be communicated in other ways. For example, often an important element or object is located in a specific place within the page (e.g. on the top right-hand corner of the page) to make it more visible. But if the element is at the end of the page code, although it is well-located visually, the screen reader will only be able to read it later, after having provided too much other information. As a result of these considerations, the developer's main goal of calling attention to an object is lost due to the time spent reaching it. Therefore, in order to achieve this, specific techniques can be used, such as CSS positioning properties, heading levels, hidden labels, different importance levels, and so forth.

Another limit of using a screen reader is related to formatting features. Often, font styles such as bold, underlined, or italic are applied to meaningful parts of the page, in order to catch reader's attention. Unfortunately, visual attributes are not captured by the screen reader at present. Assistive technologies should take this aspect into account for HTML pages as well and not only for doc or rtf documents. However, for the moment Web page developers should keep this drawback in mind. Other issues affecting aural layout are speech synthesizer pronunciation and intonation. Pronunciation aspects occur especially when reading abbreviations or foreign words. Tags structuring a phrase should be used to improve the synthesizer's pronunciation (e.g. abbr, acronym). For better speech intonation the punctuation should be used carefully and appropriately. Lastly, in order to improve the dialogue with the blind individual, the user interface should be equipped with event/alert sounds. Often a sound can communicate a written message more efficiently. Therefore, sound should be considered early in the UI design process.

In particular, aspects which developers should carefully take into account when designing search engine UIs, are:

- Interaction with search elements. In order to perform a search quickly, it is very important for users to easily identify the search box and buttons, the configuration options (language, advanced search). Thus, specific UI design features should be considered for users who interact by assistive device.

- Results. Since the main purpose of a search engine is to retrieve information, it is crucial to clearly highlight the search output (i.e. number of results) and make the result more usable (i.e. understandable and quick to visit).

- Identification of sponsored links. It is useful to clearly indicate sponsored links for easy visual identification as well as aural recognition. 
- Faster navigation. In order to improve UI usability, several specific features should be applied to the interface. Since features for a visual layout differ greatly from those for aural perception, developers should also take into account the interaction via keyboard.

In conclusion, there are several differences between visual layout and aural perception due to the way the screen reader works. Designers should apply specific guidelines and appropriate design based on the interface's purpose as well as on the need for assistive technologies. They should concentrate on the best possible design in order to improve interface usability for both visual layout and hearing navigation.

\subsection{Perception of Search Engine UIs: an example}

This section presents an example of the user interface of a wellknown search engine and shows an interaction using the JAWS screen reader. We chose Google because the graphic interface is simple, and is widely used by the blind in Italy [2].

As an example, suppose we wish to look for accessibility and usability resources. So, after having loaded the first page of Google (http://www.google.com), we type the string "accessibility usability" (without the quotation marks) and then press the "Google Search" button. The screenshot of the interface is shown in Figure 1.

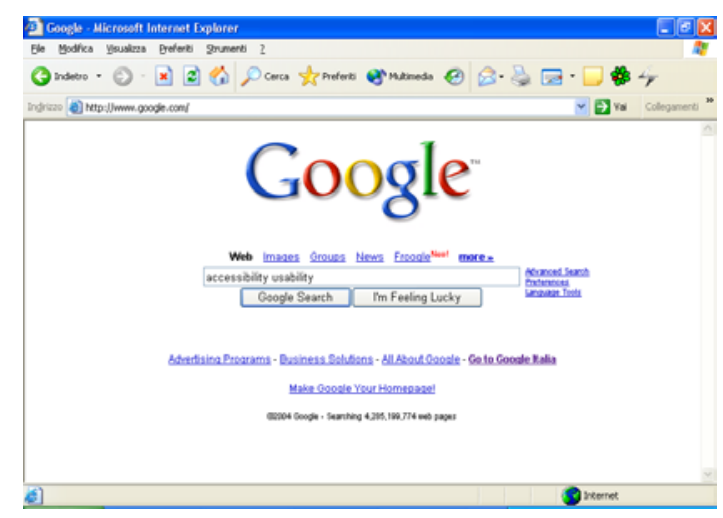

Figure 1 - Google: UI for simple search

The interface is presented as a compact screenshot where all elements are properly arranged in groups. Thus the user has an overview of the entire page contents; besides, thanks to their position, the edit field, search button and search options are all easily recognized. Unfortunately, with a screen reader it is not possible to perceive this overview and these main elements in the same way as a visual layout.

Figure 2 shows how the same page is read by the screen reader JAWS.

Google
Google
Link Web
Link Images
Link Groups

Link News
Link FroogleNew!
Link more »
Edit accessibility usability
Google Search Button
I'm Feeling Lucky Button
Link Advanced Search
Link Preferences
Link Language Tools
Link Advertising Programs -
Link Business Solutions -
Link All About Google -
Link Go to Google Italia
Link Make Google Your Homepage!
C2004 Google - Searching 4,285,199,774 web pages

Figure 2 - Google: screen reader rendering of UI for simple search (texts in italics are read but not actually displayed on the screen)

The screen reader serializes each element - link, button, field, cell and so on - so that even a simple user interface becomes particularly long and tedious. The links composing the navigation bar make reading the page more difficult; even if the search box is placed under the navigation bar, reading the page in a serial way using arrow keys, various links must be heard before reaching the box. For this reason, the navigation bar is a critical matter in interface design. Now let us consider the result page obtained from the query, as shown in Fig. 3.

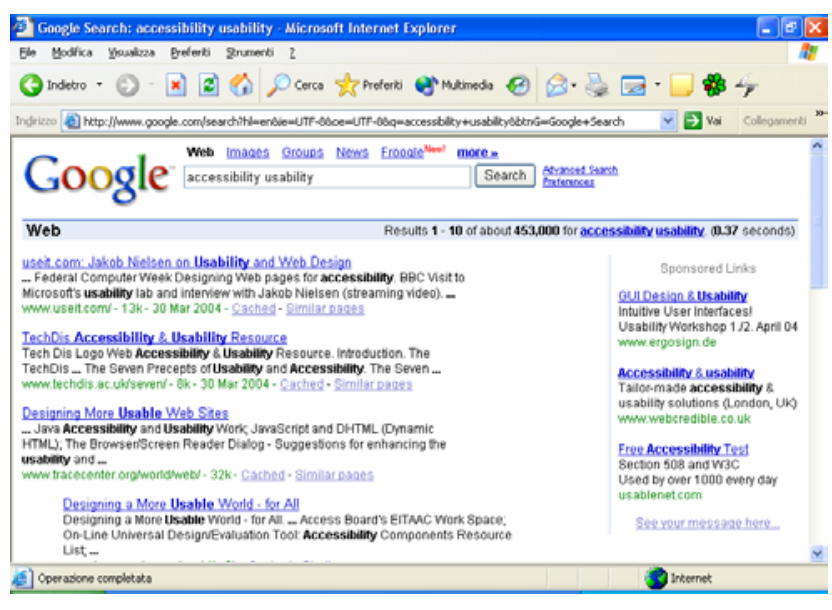

Figure 3 - Google: results page

A particular point to be considered is the "repetition of links" for each result. More precisely, we are referring to links such as "cached" and "similar pages". In fact, for each result a short summary is shown, which links the entire result page, as well as two additional links respectively to access the cache version (in case the page address is changed or the page disappeared) or for 
the retrieval of similar pages. When a blind user moves by Tab key, she/he is obliged to listen to them over and over again, making the navigation rather boring. Instead a lower tabulating value should be assigned to all secondary links; thus, moving by Tab key the main result links are visited earlier, skipping all the links of minor importance. Figure 4 illustrates how the screen reader interprets the results found by the search engine. Looking at the page, sighted users can read the results retrieved by the search engine very quickly: how many results have been found; which are the text portions containing the desired string; which are the Sponsored Links; and so forth.

Google Search: accessibility usability

Link Go to Google Home

Link Web

Link Images

Link Groups

Link News

Link FroogleNew!

Link more »

Edit accessibility usability

Search Button

Link Advanced Search

Link Preferences

Web

Results 1 - 10 of about 453,000 for accessibility usability. (0.37 seconds)

Table with 4 columns and 6 rows

Sponsored Links

Link GUI Design \& Usability

Intuitive User Interfaces!

Usability Workshop 1./2. April 04

www.ergosign.de

$\cdots$

Link See your message here...

table end

Link useit.com: Jakob Nielsen on Usability and Web Design ...

Federal Computer Week Designing Web pages for accessibility. BBC Visit to Microsoft's usability lab and interview with Jakob Nielsen (streaming video).

$\cdots$

www.useit.com/ - 13k - 30 Mar 2004 -

Link Cached -

Link Similar pages
Link TechDis Accessibility \& Usability Resource

Tech Dis Logo Web Accessibility \& Usability Resource. Introduction. The TechDis ... The Seven Precepts of Usability and Accessibility. The Seven ...

www.techdis.ac.uk/seven/ - 8k - 30 Mar 2004 -

Link Cached -

Link Similar pages

Link Designing More Usable Web Sites ...

[Other results...]

...

Result Page:

1

Link 2

Link 3

Link 4

...

Link Next

Edit accessibility usability

Search Button

Link Search within results |

$\ldots$

Link All About Google

C2004 Google

Figure 4 - Google: result page perceived using the screen reader

When the page content is read by voice synthesizer, it is not easy to skip information and links which are not closely related to the results of the search. As we see in Figure 4, before reading the number of the results found, the screen reader announces all the navigation bar links ("Go to Google Home"..."more»"), the text box containing the keywords, then other links related to search options ("Advanced Search", etc.); and finally the user can "hear" the number of results found. Hence, reading the result page in this way is tedious for a user who navigates by keyboard and accesses information by voice synthesizer (or Braille display). These negative issues can be reduced if the layout is properly designed. For this specific example, in order to improve navigability, developers should keep in mind:

- Use a heading level corresponding to the number of results "Results 1 - 10 of about 453,000 for accessibility usability" so that by means of the " $h$ " key the user can rapidly reach the page area containing the results.

- Locate the table containing sponsored links in a different place on the page. Visually it is placed on the right, but in the html code it is positioned before the results links. The same visual layout can be obtained using specific techniques by which the block can be placed in a specific place on the page (on the right) and arranging it sequentially after the effective results links. Furthermore, if the developer adds the 
attribute summary "sponsored links" to the table, users are able find it quickly by pressing the " $\mathrm{t}$ " key.

- Give a higher level to result links than other elements, such as navigation links, edit fields and buttons, and also for the specific links "Cached" and "Similar pages". In this way, moving by Tab key the user visits the result link titles first; consequently the links "Cached" and "Similar pages" are skipped: if the user wants to visit them, he/she can move by down-arrow key (to read sequentially).

- Put the links pointing to the other results pages - "1", "2", " 3 ", and so on - at the end of the results list, rather than above it; then, add an access key $(1,2,3 \ldots)$ to allow users to activate the corresponding link very quickly.

- Add links "previous" and "next", with access key "p" and "n", to navigate quickly among results pages.

However, considering that Google furnishes a very simple user interface, we also analyzed the Yahoo interface (http://www.yahoo.com/), which has a much more complex layout. As shown in Figure 5 the home page is crowded with elements (links, text boxes, image maps, etc.).

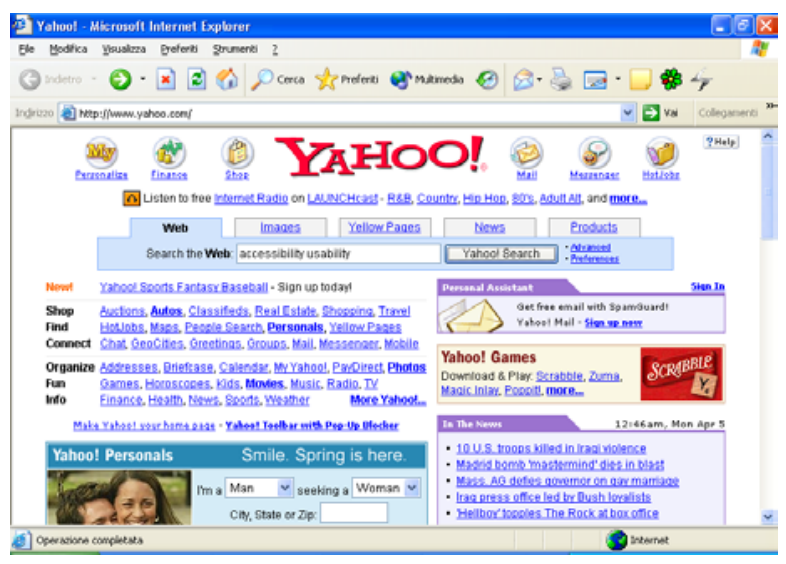

Figure 5 - Yahoo: home page

In this case, the layout is more difficult not only for visuallyimpaired users but sometimes for an unskilled person as well. When the home page is loaded, JAWS recognizes 283 links; before reading the search edit field, 23 links are read, including image map links. Figure 6 shows the results of typing in the terms "accessibility" and "usability".

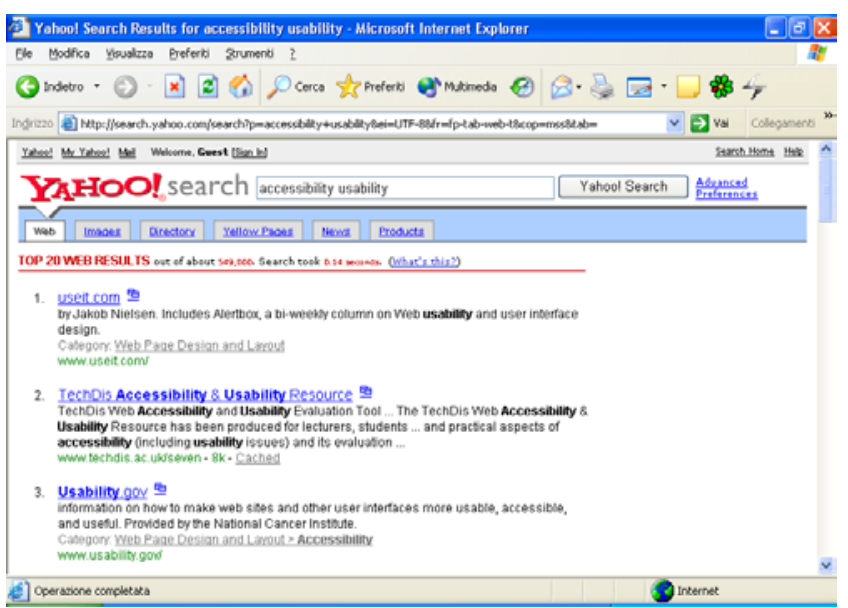

Figure 6 - Yahoo: results page

Although the visual layout is fairly simple and easy to read, perception via voice synthesizer is much more difficult. The results are arranged in a list which is useful for reading by screen reader, but it contains too many items (20). In addition, as in the Google, secondary links such as "Open this result in new window", "cached" and "Category: XXX" are present.

In conclusion, accessing Yahoo by means of a screen reader is more difficult than using Google, because both the visual layout and the structure are more complex.

\section{Proposed Guidelines for Search Engine UIs}

The developer should be aware of how the screen reader handles the Web page layout, and how blind or visually-impaired users perceive page content and interact with the interface. In this section, we propose a set of guidelines for improving the usability of a search engine user interface. The main issues in UI design are:

A. Page content serialization. The JAWS screen reader takes the page source and serializes its content (link, edit field, button, cell, and so on). Also frames or blocks $<\operatorname{div}>$ are lined up, without taking into account specific positions assigned by CSS properties. Basically, JAWS reads the code as it was written and lines up the page content in the form of a single column. Thus, the order in which the blocks $<$ div $>$ and the frames are written is very important.

B. Navigation by Tab key and special commands. It is important to remember that a blind user usually prefers to visit the page link by link (by Tab key) or using special commands in order to move quickly around the pages. Hence, it is important to favor navigation via keyboard by assigning a scale of importance to the links, applying shortcuts to main elements, using specific tags such as $<\mathrm{Hn}>$, and so on. Furthermore, many special screen reader commands operate well only if the developer has applied specific tags or attributes, or appropriate criteria have been followed.

C. What is offered by a visual layout differs from one provided for aural perception. Often when developers design a Web page they provide some useful information by means of visual features, such as position, color, separating blank spaces, formatting features, and so forth. For instance, some secondary information is put on the side so that users can recognize it immediately. It is important to provide the 
same "message" to a blind user by another means (e.g. using a table, a heading, a hidden label, etc.).

Considering all the above issues as well as accessibility and usability difficulties resulting from the screen reader, we propose that the following principles be considered in the design process of a search engine interface layout:

1. Easy location and labeling of edit field and search options. Place edit fields, option buttons and any other search element at the top of the Web page; avoid secondary elements (links, texts, banner frames, etc.). To place an object in a specific position of the visual layout, use the position CSS properties. Be careful to correctly match $<$ label for $>$ with input elements, and put labels above or to the left of the input element, rather than below.

2. Highlighting the search result. Use a heading level (i.e. $<\mathrm{h} 1>$ or $<\mathrm{h} 2>\ldots<\mathrm{h} 6>$ ) at the beginning of the result list; if possible this heading element should be the first in the page source. If a table is used to format the results, a summary attribute such as "Results of the research: xxx results found" or "No results found" should be assigned. In addition, the number of the current page vs the total number of pages should be clearly indicated (e.g. $x$ of $y$ found).

3. Arranging the results. Put the list of the result links with their summary, just after the search result notification (nothing else should be located in the middle). Create the list by applying $<\mathrm{ul}>$ or $<\mathrm{ol}>$ elements; each item on the list must be a single result. Thanks to this feature, the screen reader informs the user of the number of items; the user is then able to skip quickly item by item. Besides, the page should not contain too many results; an appropriate number would be ten items.

4. Recognizing sponsored links. Keep the sponsored links separate from the other results. Thus, put them in a clearly labelled separated table (e.g. "sponsored links" summary attribute), and insert the table code after the results list in the page source; to locate sponsored links on the right side - or in another specific place - use the CSS properties.

5. Adding navigation and help links. Locate the links pointing to result pages at the end of the list (and not before). This allows users to read first the current results (summaries and links) and then the pointers to next results; this is important when users move by arrow keys (i.e. in a sequential manner). Furthermore, it would be useful to add help or navigation links (in this case hidden links) for moving around the page such as "skip to results", "go to search edit field", and "go to result page".

6. Navigating more quickly. Assign a scale of importance (i.e. by the tabindex attribute) so users can reach the most important elements quickly. In the first search page, higher values should be assigned to edit field and search options; whereas in the result page the higher values should be given to result links. A lower value should be assigned to secondary links if present (such as "cached" or "similar pages"). Furthermore, shortcuts may be associated with search elements (text box, buttons) and links to pages of results (e.g. accesskey="1", or "2", etc.).

7. Alerting by sound. Different sounds for different events should provide useful information for blind users. For instance, two different sounds may be used for indicate the success (at least one result) or failure (no result) of the search. However, a more complex sound assignment could be applied.

8. CCS2 aural style sheets. Web designers should use aural style sheets provided by CSS2 specification for making web contents more usable and accessible to bind people. At the same time, browsers and screen readers must be able to interpret aural CSS properties.

\section{CONCLUSION}

Considering the usefulness of search tools, it is very important to make them accessible to and usable by anyone, regardless of their physical condition or environment. Accessibility and usability are essential for those who use assistive technology to navigate the Internet and search for information. In this paper we discuss accessibility and usability of search engine interfaces from the point of view of sightless users. First we described how the blind experience web searches; we then analyzed a concrete example of a search engine which offers fairly simple interfaces in terms of the elements contained in the layout. In the case of web directories when numerous services are offered and/or publicized aural navigation becomes even more complicated. Based on these considerations we proposed some guidelines for improving interface design. Considering the importance of simplifying disabled-computer interaction, search engine companies should make an effort to modify interface layout and HTML source in order to reach a greater number of users.

\section{REFERENCES}

[1] Andronico P., Buzzi M., Leporini B. (2004) Can I find what I'm looking for? (poster). WWW2004 Conference NY, USA.

[2] Buzzi M., Andronico P., Leporini B. (2004) Accessibility and Usability of Search Engine Interfaces: Preliminary Testing. To appear in Proc. of 8th ERCIM UI4ALL Workshop.

[3] Brajnik G. (2004) Achieving Universal Web Access through Specialized User Interfaces.

[4] Craven, J., Brophy, P. Non-visual access to the digital library: the use of digital library interfaces by blind and visually-impaired people. Technical report, Manchester: Centre for Research in Library and Information Management, 2003. Library and Information Commission Research Report 145.

[5] Damery E. (2003) JAWS for Windows version 4.51. New features and accessing the internet. Proceedings of 18th Technology and Persons with Disabilities Conference (CSUN), Los Angeles, California, USA.

[6] Ferworn, A., Bodner, R., Chignell, M. H. (2000), Auditory WWW Search Tools. In proceedings of the Int. Conference on Auditory Display. http://www.icad.org/websiteV2.0/Conferences/ICAD2000/P DFs/FerwornBodnerChignell.pdf

[7] JAWS for Windows, Freedom Scientific. http://www.freedomscientific.com

[8] Leporini, B., Paternò, F. (2002), Criteria for Usability of Accessible Web Sites. In proceedings of the 7th ERCIM Workshop "User Interfaces for All", Paris (Chantilly), FR, 23-25 Oct 2002, "Universal Access: Theoretical 
Perspectives, Practice, and Experience" published by Springer-Verlag, Lecture Notes in Computer Science n. 2615 (2003), pp. 43-55.

[9] Leporini, B., Paternò, F. (2003), Increasing Usability when Interacting through Screen Readers. Springer International Journal Universal Access in the Information Society (UAIS), Special Issue on "Guidelines, Standards, Methods and Processes for Software Accessibility".

[10] Marti A. Hearst (1999), User Interfaces and Visualization, In Modern Information Retrieval by Ricardo Baeza-Yates and Berthier Ribeiro-Neto, by Addison Wesley, pp.257-323.

[11] Nicolle, C., Abascal J. (2001) Inclusive design guidelines for HCI, p. 285, Taylor \& Francis, 2001.
[12] Scapin, D.L., Leulier, C., Vanderdonckt, J., Mariage, C., Bastien, Ch., Farenc, Ch., Palanque, Ph., and Bastide, R. (2000) A Framework for Organizing Web Usability Guidelines, in Ph. Kortum and E. Kudzinger (eds.). Proc. of 6th Conf. on Human Factors and the Web. http://www.tri.sbc.com/hfweb/scapin/Scapin.html

[13] Schmetzke, A., (2002) The Accessibility of Online Library Resources for People with Print Disabilities: Research and Strategies for Change. In proc. of 8th International Conf. Computers Helping People with Special Needs, pp. 390-397.

[14] W3C. Web Accessibility Initiative. http://www.w3.org/wai

[15] Section 508 standards. http://www.section508.gov 